\title{
Initiation and arrest of earthquake ruptures due to elongated overstressed regions
}

\author{
Martin Galis ${ }^{\oplus}, 1,2$ Jean-Paul Ampuero, ${ }^{3}$ P. Martin Mai ${ }^{4}$ and Jozef Kristek ${ }^{\oplus 1,2}$ \\ ${ }^{1}$ Faculty of Mathematics, Physics and Informatics, Comenius University in Bratislava, 842 48, Bratislava, Slovakia. E-mail: martin.galis@kaust.edu.sa \\ ${ }^{2}$ Earth Science Institute, Slovak Academy of Sciences, 84005 Bratislava, Slovakia \\ ${ }^{3}$ Université Côte d'Azur, IRD, CNRS, Observatoire de la Côte d'Azur, Géoazur, 06560 Valbonne, France \\ ${ }^{4}$ Division of Physical Sciences and Engineering, King Abdullah University of Science and Technology, 23955-6900 Thuwal, Saudi Arabia
}

Accepted 2019 February 13. Received 2019 February 8; in original form 2018 October 8

\begin{abstract}
S U M MAR Y
The initiation of natural and induced earthquakes is promoted in fault areas where shear stress is close to fault strength. In many real-world situations, these overstressed fault areas (or 'asperities') are very elongated; for example, in the case of a fault intersecting a reservoir subject to fluid-injection, or the stress concentration along the bottom of a seismogenic zone induced by deep fault creep. Theoretical estimates of the minimum overstressed asperity size leading to runaway rupture and of the final size of self-arrested ruptures are only available for 2-D problems and for 3-D problems with an asperity aspect ratio close to one. In this study, we determine how the nucleation of ruptures on elongated asperities, and their ensuing arrest, depends on the size and aspect ratio of the asperity and on the background stress. Based on a systematic set of 3-D dynamic rupture simulations assuming linear slip-weakening friction, we find that if the shortest asperity side is smaller than the 2-D critical length, the problem effectively reduces to a 2-D problem in which rupture nucleation and arrest are controlled by the shortest length of the asperity. Otherwise, nucleation and rupture arrest are controlled by the asperity area, with a minor exception: for asperities with shortest side slightly larger than the 2-D critical length, arrested ruptures are smaller than predicted by the asperity area. The fact that rupture arrest is dominantly controlled by area, even for elongated asperities, corroborates the finding that observed maximum magnitudes of earthquakes induced by fluid injection are consistent with the theoretical relation between the magnitude of the largest self-arrested rupture and the injected volume. In the context of induced seismicity, our simulations provide plausible scenarios that could be either favourable or challenging for traffic light systems and provide mechanical insights into the conditions leading to these situations.
\end{abstract}

Key words: Numerical modelling; Earthquake dynamics; Induced seismicity.

\section{INTRODUCTION}

A better understanding of what controls nucleation and arrest (and thus magnitude) of earthquake ruptures is naturally important. Although theoretical models and knowledge of model parameters will probably never be accurate enough to predict when earthquakes will occur and how large they will be, analysis of theoretical models provides insight into which parameters control nucleation, growth and arrest of ruptures. Such knowledge may be particularly helpful in the context of induced seismicity, where human-controlled fluid injection is believed to be responsible for triggering earthquakes (e.g. Ellsworth 2013) and measures to mitigate or avoid their hazard are being sought.
In this paper, we distinguish three classes of ruptures: runaway ruptures, self-arrested ruptures and asperity-confined ruptures. Asperity-confined ruptures cause slip only within the prestressed asperity. Self-arrested ruptures stop spontaneously (without encountering a barrier) at a finite distance from the pre-stressed asperity. Runaway ruptures propagate across the entire fault and can be stopped only by a barrier caused by stress or strength heterogeneity or by the fault geometry. Unless specified otherwise, we will use the term arrested rupture to encompass both self-arrested and asperity-confined ruptures. We will also refer to them as ruptures arrested outside or within the asperity, respectively, to emphasize explicitly the differences between arrested ruptures. 
Recently, Galis et al. (2017) applied a theoretical 3-D model of rupture arrest to induced seismicity. They considered earthquakes initiated over a limited fault area (hereafter referred to as an asperity), weakened by an increased pore fluid pressure to investigate how large ruptures may grow. They found a very good agreement between (i) their estimated magnitude of the largest arrested rupture as a function of volume of injected fluids and (ii) observed magnitudes of the largest earthquakes induced by fluid injection over a broad range of scales (from laboratory experiments to large field operations). The largest arrested rupture is defined as the largest rupture that stops spontaneously by a lack of available elastic energy, rather than by encountering a strong barrier. Larger events, the so-called runaway ruptures that propagate until stopped by a sufficiently strong barrier are still possible. In their study, Galis et al. (2017) only considered asperities of aspect ratio close to 1. However, reservoirs are often much thinner in the vertical direction than in the horizontal direction, thus their intersection with sub-vertical faults is elongated in the sub-horizontal direction, which leads to asperity aspect ratios far from 1 (Fig. 1a).

Elongated overstressed regions are also important for natural earthquakes, in particular for ruptures nucleated by stress concentrations along the bottom of the seismogenic zone, where stresses are concentrated by aseismic slip on the deeper portions of the fault (Fig. 1b). The presence of stress concentrations near transitions of fault rheology are a mathematical result of the theory of dislocations in elastic media (e.g. Kato 2012; Bruhat \& Segall 2017) and are a typical finding in interseismic stress calculations based on geodetically derived seismic coupling maps (Ader et al. 2012) or long-term slip rates (Mildon et al. 2017). Ruptures that nucleated on deep stress concentrations may remain confined at depth without breaking the entire fault width like, for example, the 2015 M7.8 Gorkha, Nepal earthquake (Avouac et al. 2015; Michel et al. 2017). Such partial ruptures may be examples of self-arrested ruptures that precede a larger event (Galis et al. 2017). Stress concentrations play an important role also in some induced seismicity situations. Notably, in producing gas fields, peaked stresses can develop along the top or bottom of the intersection between the reservoir and a fault due to the effect of differential compaction between reservoir compartments that are offset by the fault (Mulder 2003; Buijze et al. 2017; Van Wees et al. 2017).

Motivated by these considerations, our study focuses on the nucleation and arrest of earthquake ruptures due to elongated asperities. Our results extend and complement previous research on rupture nucleation and arrest based on fracture mechanics (e.g. Ampuero et al. 2006; Ripperger et al. 2007; Galis et al. 2015) including those developed in the context of induced seismicity but limited to 2-D (Garagash \& Germanovich 2012; Dempsey \& Suckale 2016; Azad et al. 2017) or to 3-D with asperity aspect ratio near 1 (Galis et al. 2017).

The nucleation and arrest of ruptures due to asperities with aspect ratio close to one has been shown to be controlled by asperity area (Galis et al. 2015, 2017). However, in the limit of an asperity with infinite or zero aspect ratio, a 3-D problem reduces to a 2-D problem, which is controlled by asperity length. Previous works studying the conditions leading to the onset of slip instabilities in 3-D (Uenishi 2009, 2018) already reported such transition, but did not examine whether the resulting rupture is self-arrested or runaway. Therefore, although the ruptured area of arrested ruptures and the conditions leading to runaway ruptures for elongated asperities have not yet been described by theoretical/analytical solutions (at least we are not aware of it), we expect these rupture properties to display a transition from an area-controlled behaviour to a length-controlled behaviour.
Here, we develop a synoptic understanding of the propagation and arrest of ruptures nucleated by elongated asperities based on numerical simulations and theoretical developments. The remainder of this paper is organized as follows. In Sections 2 and 3 we analyse the conditions required to generate runaway ruptures and the size of arrested ruptures, respectively. In Section 4 we present the implications of our results for a specific case of asperities with fixed width or length, especially in the context of induced seismicity. In Section 5 we briefly discuss the case of oblique orientation of an asperity with respect to the initial traction. The Appendices A and $B$ present a derivation of critical lengths for runaway rupture in 2-D and their verification using numerical simulations and Appendix $\mathrm{C}$ presents a summary of equations for determining conditions for runaway ruptures and rupture arrest.

\section{NUCLEATION OF RUNAWAY RUPTURES}

Here we investigate the conditions that lead to runaway ruptures for asperities of arbitrary aspect ratio. Uenishi $(2009,2018)$ considered elliptical asperities and analysed conditions leading to selfaccelerating slip. Galis et al. (2015) studied conditions for runaway ruptures in 3-D for asperities with aspect ratio close to one. For high background stress, they found that an asperity area greater than that estimated by Uenishi $(2009,2018)$ is also sufficient to produce runaway ruptures. For lower levels of background stress, after onset a rupture either arrests spontaneously or becomes runaway; in this context, Galis et al. (2015) derived a new estimate of the critical area leading to runaway ruptures. Because of the expected transition from an area-controlled 3-D problem to length-controlled 2-D problem, we here first derive 2-D estimates of critical length that are physically consistent with the 3-D estimates of critical area by Galis et al. (2015). Then, we compare our numerical results for elongated asperities with the 2-D and 3-D theoretical estimates. Finally, we propose a general procedure to estimate whether an elongated asperity will produce a runaway or self-arrested rupture.

We followed the approach of Galis et al. (2015) and derived theoretical estimates of a 'critical length' for 2-D problems, defined as the minimum asperity length that can produce runaway ruptures. The critical length is defined as $a_{\mathrm{c}}=\max \left(a_{\mathrm{UR}}, a_{2}\right)$, where $a_{\mathrm{UR}}$ is the estimate in the so-called large-scale regime by Uenishi \& Rice (2003; eq. A14) and $a_{2}$ is our estimate in the so-called small-scale regime derived in Appendix A (eq. A13). We verified the estimate $a_{\mathrm{c}}$ using numerical simulations (Appendix B). The critical length $a_{\mathrm{c}}$ depends on strength parameter $S=\left(\tau_{s}-\tau_{0}\right) /\left(\tau_{0}-\tau_{d}\right)$, where $\tau_{0}$ is the initial background shear traction, and $\tau_{\mathrm{s}}$ and $\tau_{\mathrm{d}}$ are static and dynamic friction strengths, respectively. For low $S$ (i.e. high background stress) the critical length $a_{\mathrm{c}}$ for mode II (in-plane) and III (anti-plane) is determined by $a_{\mathrm{UR}}$, which does not depend on $S$ (Fig. B1). For greater $S$ (i.e. low background stress), the critical length $a_{\mathrm{c}}$ is determined by our estimate $a_{2}$, which grows with increasing $S$. The transition between these two regimes occurs at $S \approx 2.75$ (see Fig. A1). This behaviour is qualitatively consistent with 3-D results by Galis et al. (2015), but in 3-D the transition between large-scale and small-scale yielding regimes occurs at $S \approx 0.75$ (see fig. $10 \mathrm{~b}$ of Galis et al. 2015). The difference has a geometrical origin: the dimension of the problem affects both the small- and large-scale yielding critical lengths. For instance, it affects the former by changing how much the two sources of stress drop (the localized load and the background uniform stress) contribute to the static stress intensity factor $K_{0}$, and how fast the 
(a) fluid injection into a reservoir

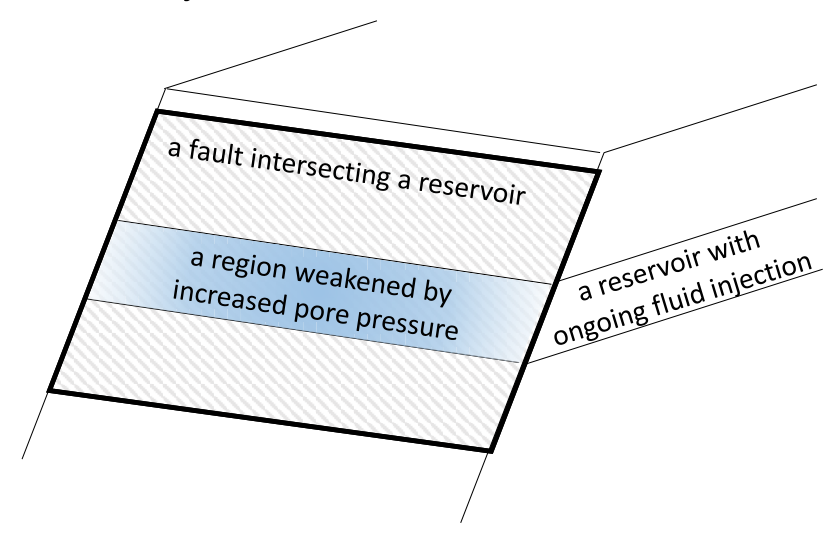

(b) a stress concentration at an interplate interface

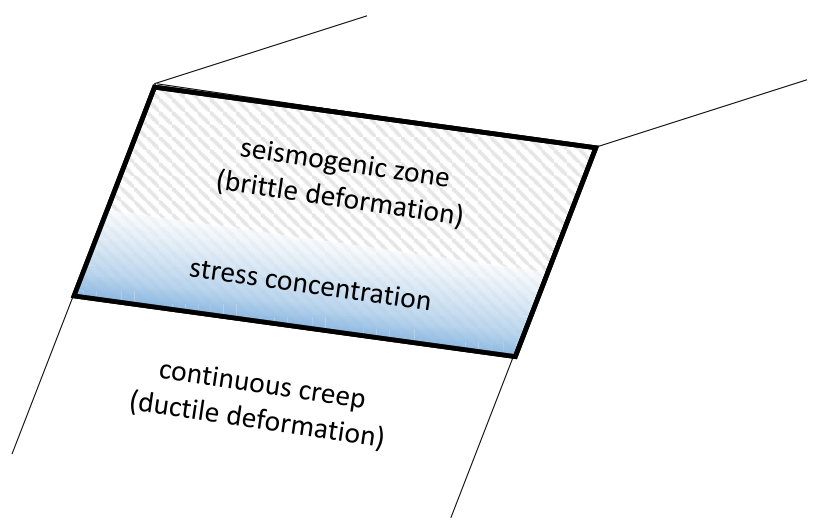

Figure 1. Two examples of situations leading to elongated overstressed asperities. (a) A fluid injection into a reservoir intersected by a fault—reservoirs are often much thinner in the vertical direction than horizontally; therefore, their intersection with a sub-vertical fault, weakened by increased pore pressure, is elongated. (b) Interplate interface — deep fault creep induces elongated stress concentrations along the bottom of the locked seismogenic zone.

localized load contribution decays with rupture length or radius. This can be appreciated more easily in the point-load approximation by comparing our eqs (A5) and (A4) of Galis et al. (2015). These geometric effects in turn result in different $S$ values in 2-D and 3-D at the transition between large- and small-scale yielding regimes.

Because there is no analytical estimate of the critical area of an elongated asperity, we performed numerical simulations with elongated asperities for a wide range of aspect ratios. For the simulations, we used the finite-element method (e.g. Moczo et al. 2014) and a regular mesh, with the fault being discretized by square elements. To achieve an exact discrete-mesh geometrical representation of the asperity, we consider a rectangular asperity. In the numerical simulations, we used the same parameters as for the verification of the 2-D estimates (Table B1). By varying the initial traction $\tau_{0}$ we obtained $S$ values of $0.1,0.5,1,1.5,2,3$ and 4 . We considered a fixed ratio between overstress and strength excess $(0.5$ per cent) and asperities with aspect ratios ranging from 1:30 to 50:1. The aspect ratio $1: 1$ corresponds to a square, $5: 1$ to a rectangle elongated in the mode II direction and 1:5 to a rectangle elongated in the mode III direction (Fig. 2a). All the results are plotted using rupture length and area normalized by $L_{\text {fric }}$ and $L_{\text {fric }}^{2}$, respectively, where $L_{\text {fric }}=\mu . D_{\mathrm{c}} /\left(\tau_{\mathrm{s}}-\tau_{\mathrm{d}}\right)$ is the characteristic length scale induced by slip-weakening friction (e.g. Ampuero et al. 2002; Dunham 2007).

The critical conditions at the transition to runaway ruptures in our simulations are identified as follows. For each individual numerical simulation, we consider an asperity with fixed size and overstress and we evaluate whether the resulting rupture propagated across the entire fault plane (runaway rupture) or whether it stopped spontaneously at a finite distance (self-arrested rupture). Our focus here is the transition to runaway ruptures, therefore, the latter option implicitly covers also cases when the rupture is arrested within the asperity. Our numerical results are summarized in Fig. 2(b). To visualize the critical conditions, the empty symbols depict the largest asperity, for a specific aspect ratio, producing arrested rupture and filled symbols denote the smallest asperity producing a runaway rupture. For better visualization of the transition, we considered asperities with aspect ratios close to several predefined/discrete values (1:30, 1:25, etc). For cases with $S \leq 2$, a $30 \mathrm{~km} \times 15 \mathrm{~km}$ fault is sufficient to contain the largest arrested ruptures; however, for $S=3$ and $S=4$ we used faults with dimensions up to $60 \mathrm{~km} \times$
$30 \mathrm{~km}$ to accommodate not only the largest arrested ruptures but also asperities with extreme aspect ratios.

Our simulations confirm that the transition to runaway ruptures follows mainly two regimes depending on asperity aspect ratio. For aspect ratio $1: 1$, the numerical results are consistent with the theoretical criterion based on critical area by Galis et al. (2015; bold lines in Fig. 2b). For intermediate aspect ratios (e.g. ranging from 1:4 to 8:1 for $S=2$ ), an area-controlled regime prevails: the runaway transition is controlled by the same critical area criterion as for a square asperity (1:1). For more elongated asperities (e.g. aspect ratios smaller than 1:8 and larger than 14:1 for $S=2$ ), we find a length-controlled regime: the runaway transition occurs when the narrower side of the asperity exceeds the 2-D critical length (Appendix A) regardless of asperity area. The critical lengths are consistent with the corresponding 2-D theoretical estimates in both mode II and III directions (thin lines in Fig. 2b). The range of aspect ratios in the area-controlled regime grows with increasing $S$, especially when $S<2$. The area-controlled and length-controlled regimes show asymptotic behaviour; the transition between them, as a function of aspect ratio, is rather sharp for $1 \leq S \leq 2$ but more gradual for $S=0.5$. Theory predicts that in 3-D the critical area depends on $S$ if $S>0.75$ (Galis et al. 2015), whereas in 2-D the critical length grows with $S$ if $S>2.75$ (Appendix B). This explains why in Fig. 2(b), for $1 \leq S \leq 2$, the runaway transitions coalesce in the length-controlled regime (see overlapped symbols in Fig. 2b) but are separated in the area-controlled regime; and for $S=3$ and $S=4$, the runaway transitions are separated also in the lengthcontrolled regime. The deviation from the theoretical estimates for $S=0.1$ and $S=0.5$ - for which the numerical results indicate a larger critical area than the theoretical estimate - is a consequence of numerical artefacts of the rectangular asperity, reported previously by Galis et al. (2015), who observed relatively slow convergence of the critical area for the square asperity, particularly for $S<$ 0.75 (see their fig. 9). They observed the fastest convergence for an elliptical asperity with aspect ratio 4:3. However, a stair case representation of an ellipse in a regular mesh requires a very small element size for a proper geometrical representation of elongated asperities, which would lead to prohibitively large computational costs for our parametric study.

Based on these results, in particular on the agreement between theory and simulation in the dependence of the runaway transition on $S$, we conclude that the transition to runaway ruptures is controlled 
(a)
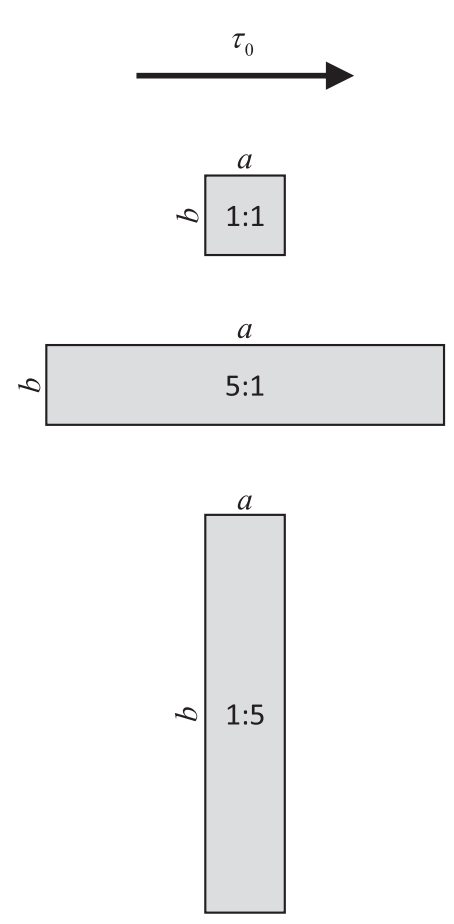

(b)

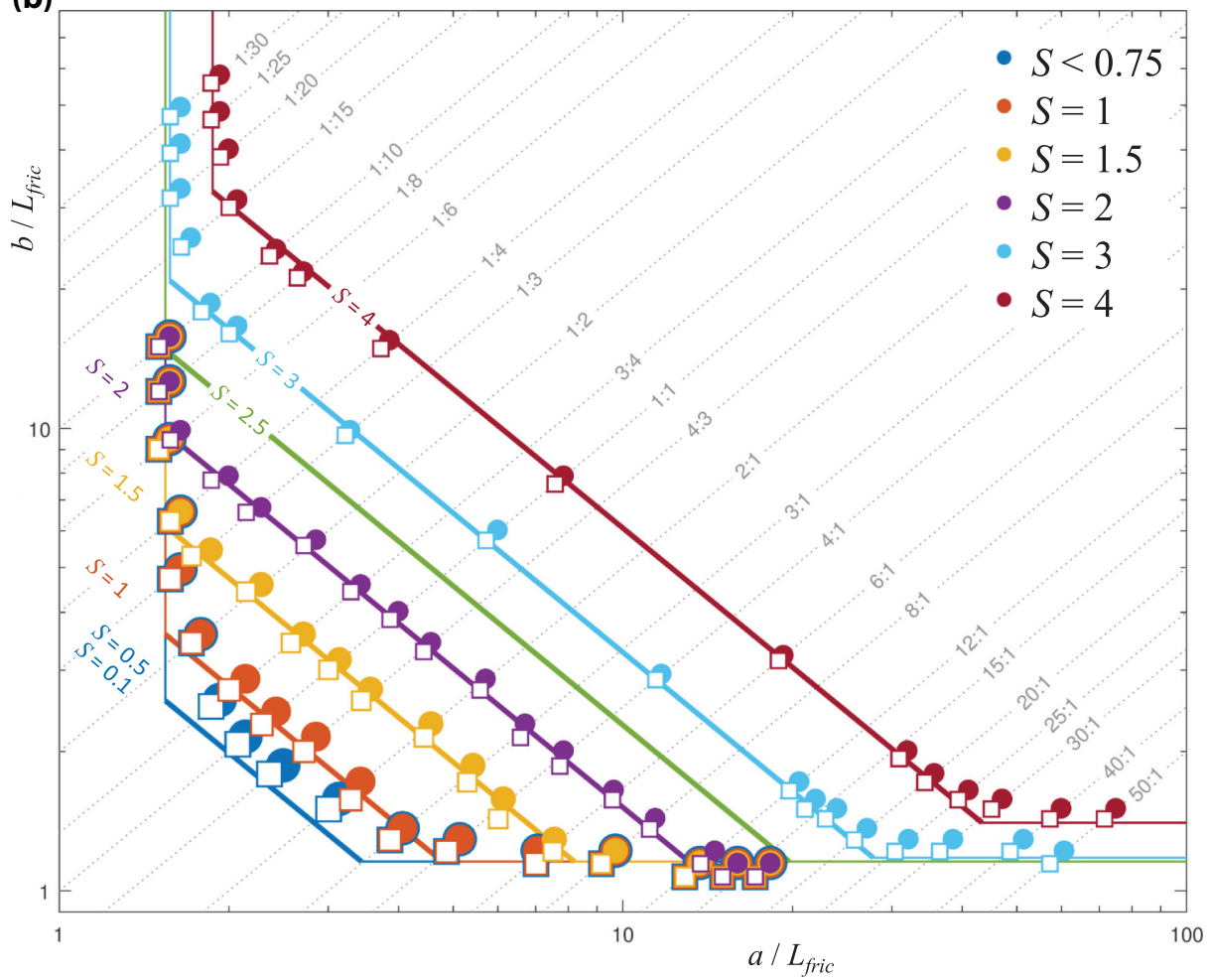

Figure 2. (a) Overstressed asperities of different shapes (top to bottom): square, elongated in mode II and in mode III directions with respect to the orientation of the initial traction, $\tau_{o}$ (arrow on top). The asperity sizes $a$ and $b$ correspond to the mode II and mode III edges of the asperity, respectively. (b) Comparison of theory and simulations of transition to runaway ruptures as a function of the dimensions of an overstressed asperity and background stress. The empty symbols denote the largest asperities, for a specific aspect ratio, still leading to arrested ruptures in our simulations; the solid symbols denote the smallest asperities leading to runaway ruptures. Results are colour-coded by their assumed value of the non-dimensional strength parameter $S$ (see legend and coloured labels). Due to varying colour and size of symbols, the overlapping cases appear to have outlines of different colours. The bold diagonal lines indicate the theoretical critical area criterion (Galis et al. 2015), thin vertical and horizontal lines our 2-D estimates of critical lengths (Appendix A). Results for $S<0.75$ are identical as illustrated by $S=0.1$ and $S=0.5$. The dotted diagonal lines are lines of equal aspect ratio (see grey labels).

either by (i) the critical area if the narrower side of the asperity is greater than the corresponding 2-D estimate of the critical length, or (ii) by the critical length. We propose the following procedure to determine whether an elongated asperity (with area $A=a \times b$, and aspect ratio $\alpha=a / b ; a$ and $b$ are the lengths of the asperity in mode II and III directions, respectively) will produce a runaway or an arrested rupture (the equations used in the procedure are also summarized in Table C1):

(1) Estimate the critical area, $A_{\text {crit }}$, following eqs (17-22) and (24-26) of Galis et al. (2015)

(2) Estimate the critical lengths $a_{\text {crit }}$ and $b_{\text {crit }}$ for modes II and III, respectively, following eqs (A13-A15) in Appendix A

(3) Ruptures are runaway if the following three conditions are met simultaneously:

$A>A_{\text {crit }}, a>a_{\text {crit }}$ and $b>b_{\text {crit }}$. Otherwise, ruptures are arrested.

These approximate conditions define each runaway boundary in Fig. 2 by three straight lines. The true boundaries may have some curvature near the transition between the area-controlled and length-controlled regimes. Such curvature is visible in Fig. 2 in the simulation results with the two largest values of $S$. For completeness, we note that the smallest and largest aspect ratios for the areacontrolled regime may be estimated by

$\alpha_{\min }=\frac{a_{\text {crit }}^{2}}{A_{\text {crit }}} \quad$ and $\quad \alpha_{\max }=\frac{A_{\text {crit }}}{b_{\text {crit }}^{2}}$.

\section{EXTENT OF ARRESTED RUPTURES}

In the previous section, we investigated the conditions leading to runaway ruptures. Now, we are interested in estimating the size of self-arrested ruptures, and in particular, its dependence on asperity aspect ratio.

Previous 2-D and 3-D results provide final rupture sizes for end member cases of asperity aspect ratios, but also suggest that the intermediate cases may be non-trivial. In 2-D, Ampuero \& BenZion (2008; their Appendix B) applied fracture mechanics theory to estimate the final length of self-arrested ruptures as a function of asperity length. In 3-D, Galis et al. (2017) used a similar approach to estimate the final rupture area. For asperities with aspect ratios close to one, they found that ruptured area is controlled by asperity area. The theoretical estimates derived by the two studies were supported by simulation results and complement each other. Considering a wider range of asperity aspect ratios in 3-D, just like for the runaway transition, we expect a transition from purely 3-D behaviour (area-controlled) for asperities with aspect ratio close to one to 2-D behaviour (length-controlled) for extremely elongated asperities. However, in 2-D, ruptures arrested outside of an asperity occur for $S>2.75$, whereas in 3-D and for asperities with aspect ratio close to one, they occur already for $S>0.75$. This suggests that aspect ratio has a more complex effect on rupture arrest than on the runaway transition. 
Our new simulations provide a comprehensive picture of how the asperity aspect ratio affects rupture arrest. To allow better coverage of the arrested ruptures, we performed additional simulations, in addition to those included in Fig. 2. The results are summarized in Fig. 3. Each row shows results for a fixed $S$ value ranging from 1 to 4 . Results for $S=0.1$ and $S=0.5$ are not shown because only ruptures arrested within an asperity or runaway ruptures exist for $S<0.75$. Overall, by comparing the numerical results with $2-\mathrm{D}$ and 3-D theoretical estimates, we identify three regimes: rupture arrest controlled by asperity length (squares), by asperity area (circles) and by neither length nor area (diamonds).

Our simulations confirm that the end-member regimes of rupture arrest are consistent with theoretical expectations. In the areacontrolled regime of rupture arrest, for all aspect ratios, we find almost perfect agreement between the numerically simulated and theoretically predicted areas of arrested ruptures (yellow circles and yellow curves, respectively, in the left-hand column of Fig. 3). In this regime, the runaway transition is also area-controlled.

In the following discussion, we limit ourselves to ruptures arrested outside an asperity. In the length-controlled regime of rupture arrest (which exists only for $S>2.75$ ), the final rupture size is consistent with the theoretical estimates for mode II and mode III depending on the orientation of the elongated asperity (squares and curves of corresponding colours in Figs $3 \mathrm{~b}$ and e). The agreement is, however, not as perfect as in the area-controlled regime. This could be affected by prevailing 3-D effects or non-zero stress drop. Although the asperities in the length-controlled regime are very elongated, they are finite and 3-D effects may still affect rupture propagation. This would imply smaller discrepancies for more elongated asperities, which are better approximations of a 2-D problem. Because we do not observe such behaviour, we conjecture that the observed deviations are caused by non-zero stress drop (i.e. $\tau_{0}-\tau_{\mathrm{d}}>0$ ). Ampuero \& Ben-Zion (2008) observed almost perfect agreement between 2-D predictions and simulations of arrest size only for $\tau_{0}-\tau_{\mathrm{d}}=0$, whereas in all our simulations $\tau_{0}-\tau_{\mathrm{d}}>0$. Finally, for all our cases in the length-controlled regime of rupture arrest, the runaway transition is also length-controlled.

The most interesting regime is the third one, which we identified for $S>1.5$ (diamonds in Fig. 3). In this non-trivial regime, the simulated arrest sizes are not consistent with theoretical predictions, even though the transition to runaway ruptures is. While the length of asperities in this group is in a range where 2-D estimates predict runaway ruptures, the asperities still produce arrested ruptures (middle column of Fig. 3). Moreover, the arrest area is smaller than that predicted by the 3-D estimate (left-hand column of Fig. 3). The fact that neither asperity area nor length describe this group sufficiently well suggests that rupture arrest may be controlled by mixed mode II + III rupture. In our simulations the asperities sides are parallel or perpendicular to the orientation of initial traction. For asperities with aspect ratio close to one or for very elongated asperities, this makes no difference because rupture nucleation and arrest are controlled by area or length, respectively. However, for the intermediate regime, the effect of area is no longer sufficient and the effect of length is not yet strong enough. Therefore, we conjecture that in this range the mixed mode II + III plays an important role in rupture nucleation and arrest. This also suggests that the role of mixed mode could be important for cases with oblique orientation between the asperity and initial traction. We will briefly discuss this aspect in Section 5.

We propose a general procedure, shown in Fig. 4, for estimating the area of arrested ruptures, $A_{\text {arr }}$, nucleated by an elongated asperity. The procedure is applicable for ruptures arrested outside or within an asperity but cannot be used to estimate the size of runaway ruptures, whose size is generally controlled by barriers and fault geometry. Note that the factor 2 used to limit the intermediate regime is an approximation based on numerical results.

\section{ASPERITY WITH FIXED WIDTH OR LENGTH}

In previous sections, we considered an asperity of a given size and investigated the rupture size it can produce. However, in nature, asperities grow in time. In a fluid-injection context, the fault region affected by fluid pressure and poroelastic stress perturbations expands as injection progresses. In a tectonic context, the spatial extent of the stress concentration near the edge of a locked fault area increases due to creep in the unlocked area. One dimension of the growing overstressed area may be constrained, for example, by the thickness of the pressurized reservoir. Having this interpretation in mind, we will now discuss the special case of an asperity with fixed width or length but increasing area, with potentially important implications for fluid injection operations.

We note that, when simulating ruptures on asperities of a given size, we have not accounted for the possibility that the asperity may have produced (seismic or aseismic) ruptures previously when it was still smaller. In particular, we neglected any stress redistribution caused by such previous events. Thus, our estimate of the size of arrested ruptures can be considered as an upper bound for a given asperity size.

The results presented in Fig. 2 are valid for any asperity. The length $a$ and width $b$ have to be compared with the critical curve corresponding to the given $S$-value. As shown in the conceptual figure (Fig. 5), if the asperity dimensions fall below the critical curve, the asperity produces an arrested rupture (either asperity-bounded or arrested outside of the asperity), otherwise it produces a runaway rupture. Faults producing arrested ruptures lead to smaller earthquake magnitudes, and thus pose less hazard than faults producing runaway ruptures. If $a$ and $b$ are far from $a_{\text {crit }}$ and $b_{\text {crit }}$, respectively, the system behaviour is relatively predictable, it is only mildly affected by small changes of $a$ or $b$. However, this picture changes if $a$ or $b$ are close to their critical values. This is better seen using examples.

We first illustrate the transition to a runaway rupture for an asperity with fixed width $b$ and growing length $a$. Increasing its area moves it along a horizontal line in Figs 2(b) and 5. If $b$ is far from $b_{\text {crit }}$, the system's behaviour is relatively predictable. On the other hand, if $b$ is close to $b_{\text {crit }}$, the system becomes very sensitive to $b$. We illustrate this behaviour using numerical simulations, described in terms of dimensional values for width and length; however, note that these are only valid for our particular choice of parameter values. We consider $S=4$ and a fixed and slightly overcritical width $b$ (corresponding to Scenario 1 in Fig. 5). Here, the critical sizes are $a_{\text {crit }}=1.31 \mathrm{~km}$ and $b_{\text {crit }}=0.98 \mathrm{~km}$. The simulation results in Fig. 6(a) show that an asperity with length $a=1.35 \mathrm{~km}$ produces ruptures bounded in all directions by the asperity. Increasing $a$ to $2.6 \mathrm{~km}$ leads to self-arrested ruptures that propagate outside the asperity, mostly in the vertical direction, while their horizontal size remains constrained by the asperity length. Their rupture size grows with growing $a$. Eventually, if $a=42 \mathrm{~km}$, above the critical length, the fault produces runaway ruptures.

We now consider an asperity with fixed length $a$ and growing width $b$. Increasing its area moves it along a vertical line in Figs 2(b) and 5 . We consider again $S=4$, set $a=42 \mathrm{~km}$ (a value producing 

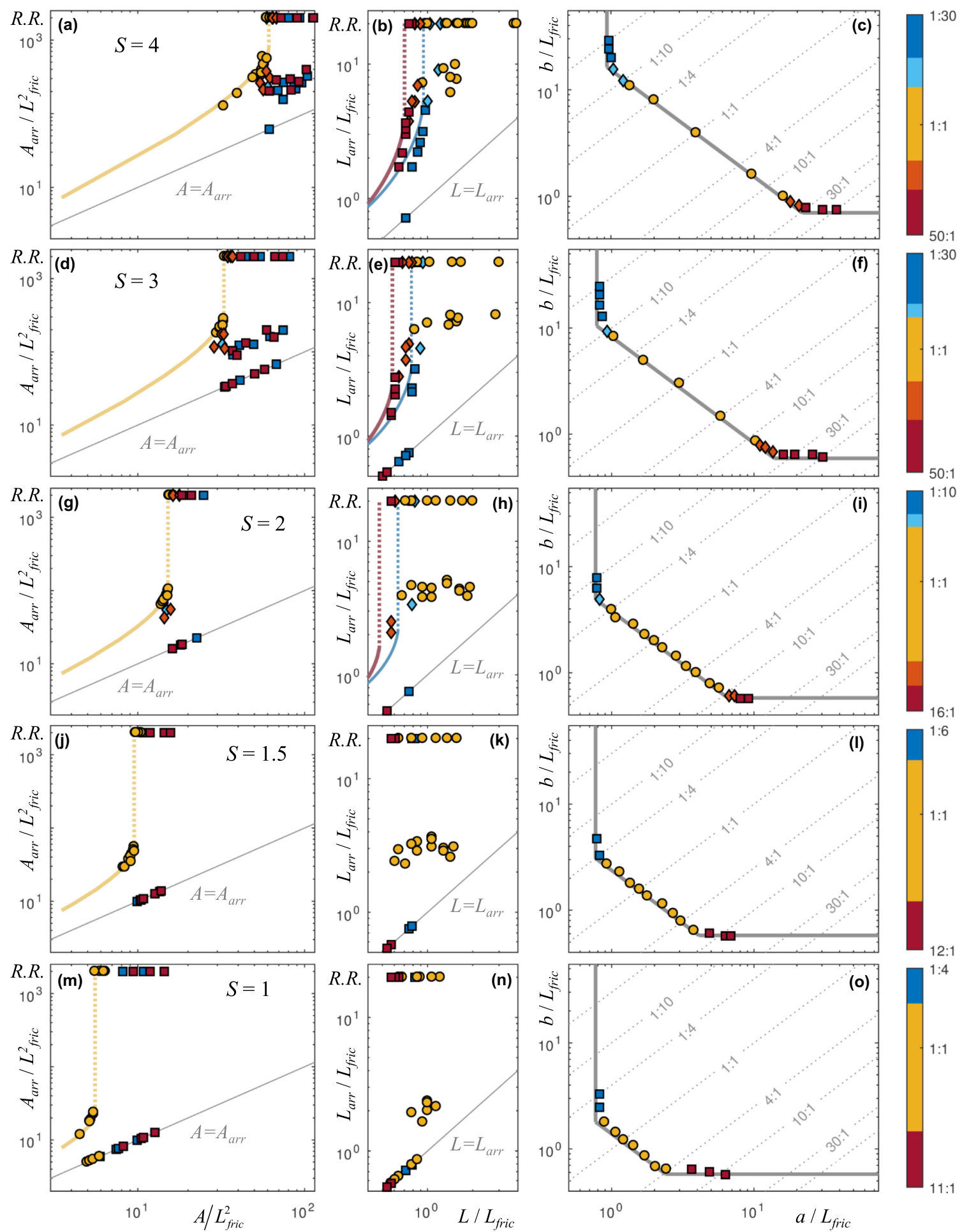

Theoretical estimates

estimate of arrested area (Galis et al, 2017)

estimates of arrested length
(Ampuero and Ben-Zion, 2008) $\left\{\begin{array}{l}\text { for mode II } \\ \text { for mode III }\end{array}\right.$

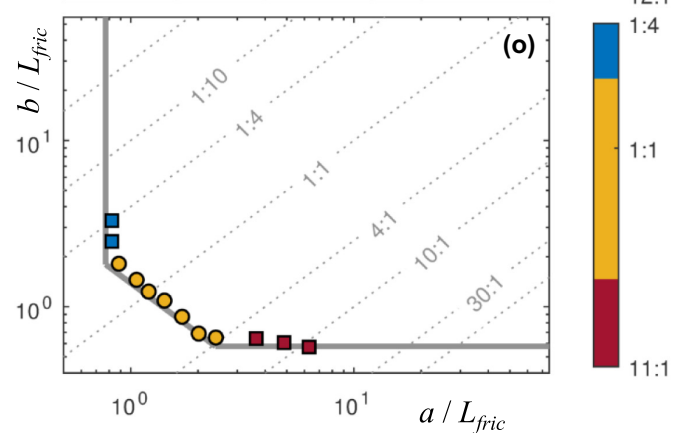

Rupture arrest controlled by

$\square$ length of asperity (group 1)

$\circ$ area of asperity (group 2)

$\diamond$ neither length nor area of asperity (group 3)

Figure 3. Left-hand column: comparison of area of arrested ruptures with theoretical estimate. Middle column: comparison of arrested length with theoretical estimates. Right-hand column: separation of the three groups in the $a-b$ plot (compare with Fig. 2b). Different symbols represent the three groups of behaviour while colour of symbols represent aspect ratios (red and blue tones depict asperities elongated in mode II and III directions, respectively). R.R. on $y$-axes denotes runaway ruptures (i.e. cases in which the entire fault ruptured). 


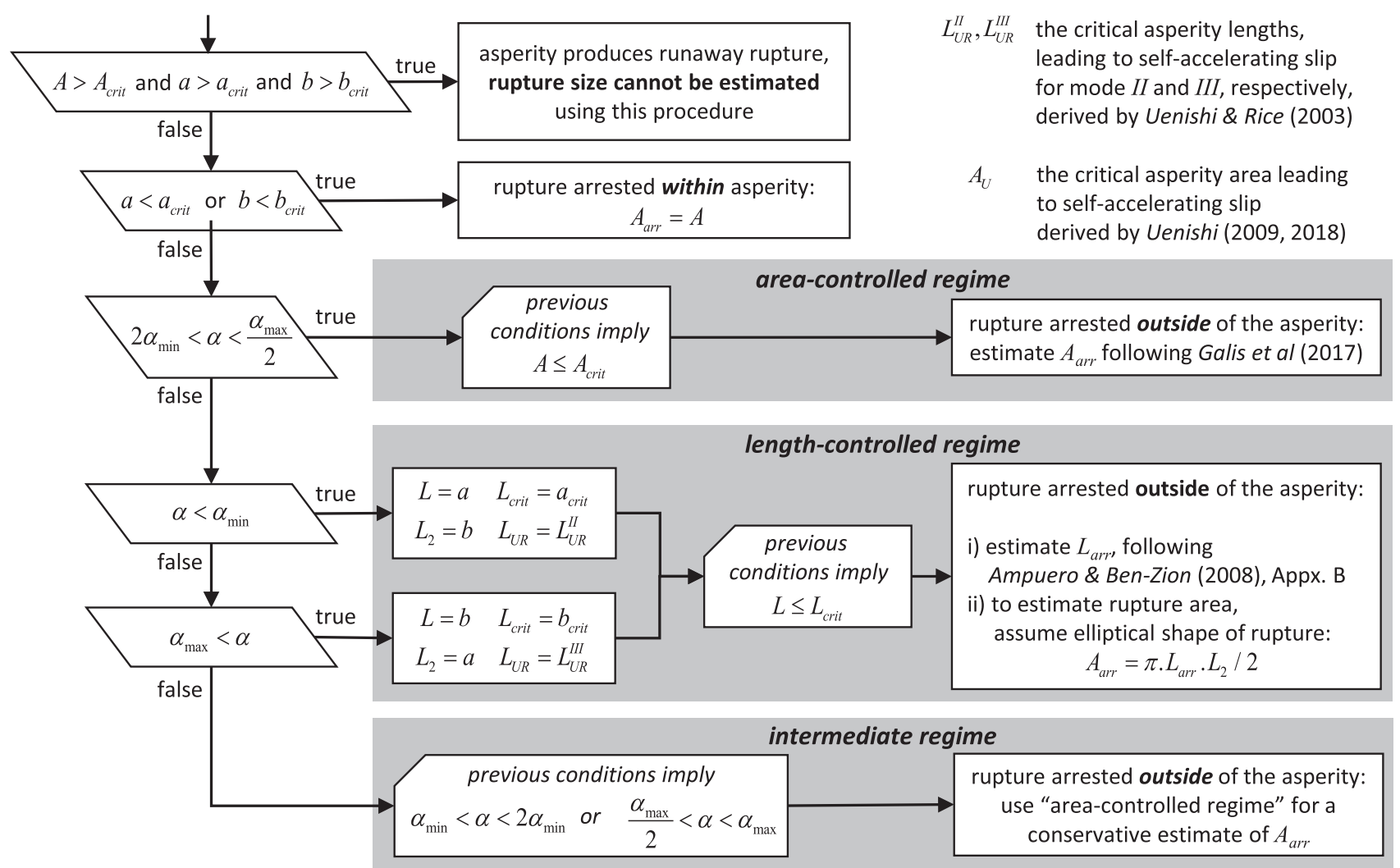

Figure 4. Procedure for estimating the area of arrested ruptures, $A_{\text {arr }}$, nucleated by an asperity with area $A=a \times b$ and aspect ratio $\alpha=a / b ; a$ and $b$ are the lengths of the asperity in mode II and III directions, respectively. An overview of the procedures for estimating rupture arrest size for area-controlled and length-controlled regimes can be found in Table $\mathrm{C} 2$.

runaway rupture) and then vary $b$ (corresponding to Scenario 2 in Fig. 5). As shown by our numerical simulations (Fig. 6b), an asperity with $b=0.75 \mathrm{~km}$ (and narrower) produces ruptures bounded by the asperity while an asperity with $b=1.05 \mathrm{~km}$ (and wider) already produces a runaway rupture. At intermediate values of $b$, ruptures propagate outside the asperity in the vertical direction and then self-arrest.

Both sets of examples can be viewed as approximations of scenarios of fluid injection into a reservoir bounded by impermeable layers and intersected by a fault. The first scenario can be seen as, for example, injection into a laterally extended reservoir with no fluid migration along the fault, that is, there is no fluid-leakage out of the reservoir, not even along the fault in the vertical or horizontal directions. Another interpretation of the first scenario is injection into a fault with only horizontal (i.e. along-strike) fluid migration. The second case corresponds to fluid-injection into a saturated reservoir with fluid migration along the fault in the vertical direction (i.e. along dip). We note that these are just example interpretations and other interpretations may exist. As indicated in Fig. 5, in the first scenario, the system produces arrested ruptures that propagate outside the asperity for a very broad range of asperity lengths ( $a$ from 1.35 to $42 \mathrm{~km}$ ). In contrast, in the second scenario this occurs only for a narrow range of asperity widths (roughly $b$ from 0.75 to $1.05 \mathrm{~km}$ ), where even relatively small changes in width are sufficient to dramatically affect the rupture behaviour. Here we considered a low initial shear stress $(S=4)$ for which arrested ruptures exist even in the length-controlled regime. However, faults with higher initial stress levels $(S<2.75)$ do not produce arrested ruptures in the length-controlled regime (Appendix A), and thus, there would be a direct transition from ruptures bounded by the asperity to runaway ruptures in the second scenario.

These idealized examples reveal important implications for traffic light systems (TLSs) used to monitor fluid-injection operations. (1) In the first scenario, regardless of the tectonic stress level, ruptures arrested outside of the asperity would happen during a relatively long period of injection with sizes growing significantly before the transition to runaway rupture occurs. This progressive evolution towards runaway rupture could potentially provide enough time for TLS to detect it and for operators to respond to prevent a runaway rupture. (2) In the second scenario and at high tectonic-stress level $(S<2.75)$, the system produces either asperity-confined or runaway ruptures. The absence of ruptures arrested outside the asperity eliminates a potential precursor to runaway ruptures, which poses a particular challenge for TLSs. (3) The sensitivity of the system to relatively mild changes of width $b$ together with higher permeability along a fault (compared to reservoir permeability) imply that the transitions from asperity-confined ruptures to ruptures self-arrested outside the asperity and then to runaway ruptures could occur faster in the second scenario than in the first one, posing another challenge for TLSs.

\section{OBLIQUE ORIENTATION OF INITIAL STRESS AND ASPERITY}

In the previous analysis, we considered asperities oriented either parallel or perpendicular to the initial shear traction (Fig. 2a). A complete analysis of cases with oblique orientation is beyond the 


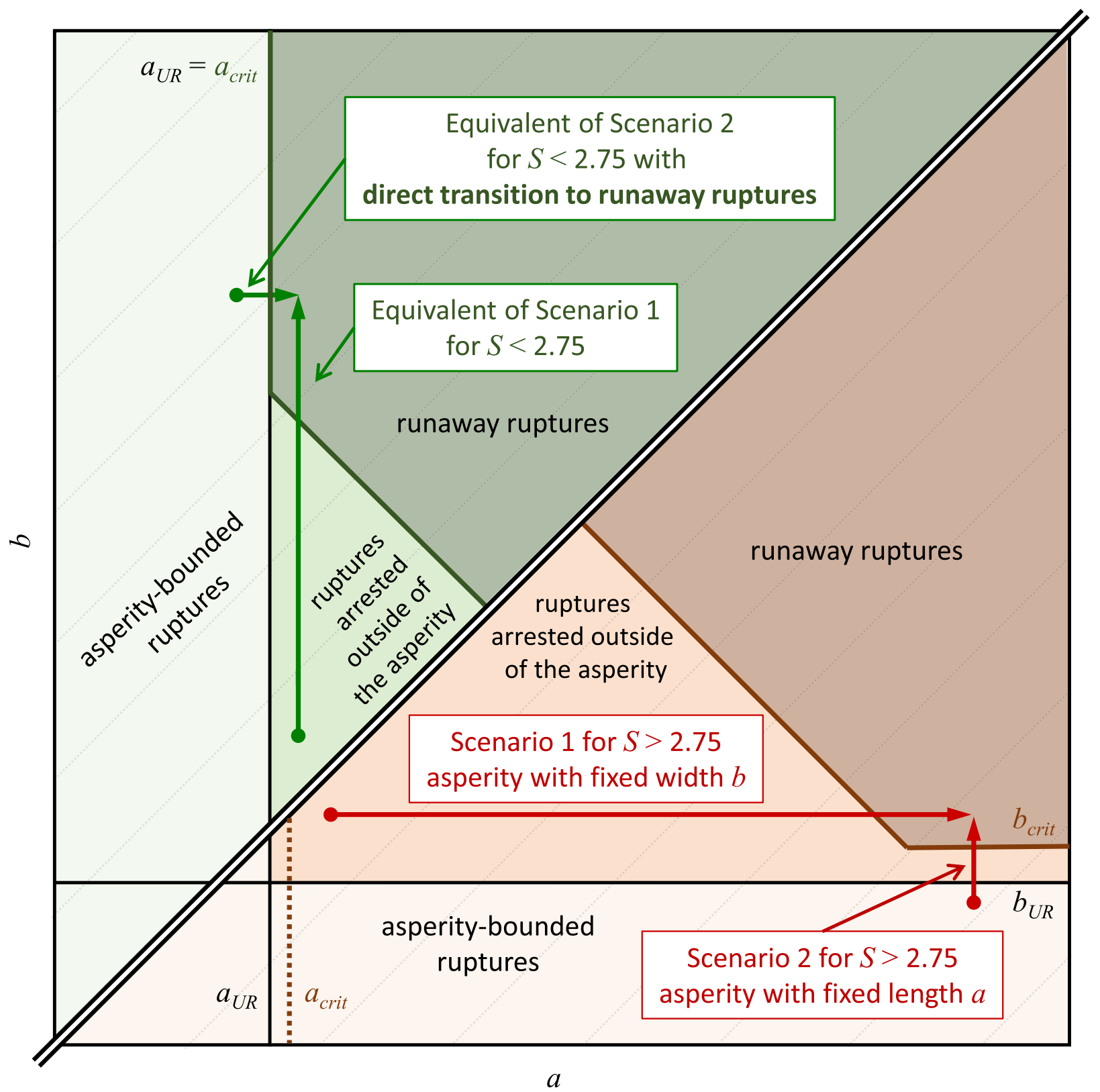

Figure 5. Dependence of rupture modes on asperity size for $S<2.75$ and aspect ratios smaller than one (upper triangle) and for $S>2.75$ and aspect ratios greater than one (bottom triangle). This compact representation of both cases exploits the quasi-symmetry of Fig. 2(b). For $S>2.75$, scenarios for $b \sim b_{\text {crit }}$ are shown (as discussed in the text). For $S<2.75$, equivalent scenarios for $a \sim a_{\text {crit }}$ are shown.

scope of this paper. However, we expect qualitatively similar results, in particular the runaway transition should also be controlled by either asperity area or length, depending on asperity aspect ratio, although possibly with different values of critical area and critical length. Ripperger et al. (2007) studied ruptures on faults with stochastic heterogeneous stress distributions, which resulted in asperities of irregular shape. They found that rupture nucleation was achieved if the radius of the inscribed circle of the irregular asperities exceeded a critical nucleation length. Similarly, for oblique stress-asperity orientations, we conjecture that runaway ruptures occur if the inscribed ellipse with major axis oriented parallel to the initial traction has major and minor axes larger than the critical lengths $a_{\text {crit }}$ and $b_{\text {crit }}$, respectively, and its area is larger than $A_{\text {crit }}$.

To test our conjecture, we performed three simulations with $S=4$ and initial traction rotated by $45^{\circ}$ with respect to the asperity (Fig. 7). In the first simulation, we set an asperity size leading to runaway rupture if $\tau_{0}$ is oriented parallel to the asperity elongation ( $a=52.5 \mathrm{~km}$ and $b=1.05 \mathrm{~km}$, i.e. aspect ratio $50: 1)$. Under oblique initial stress, the asperity is too narrow to include the critical ellipse determined by $a_{\text {crit }}$ and $b_{\text {crit }}$ (Fig. 7a) and, as expected, it produced an arrested rupture (Fig. 7b). In the second simulation we set $b=1.15 \mathrm{~km}$. In this case, the asperity is slightly narrower than the critical ellipse and also produced an arrested rupture. In 

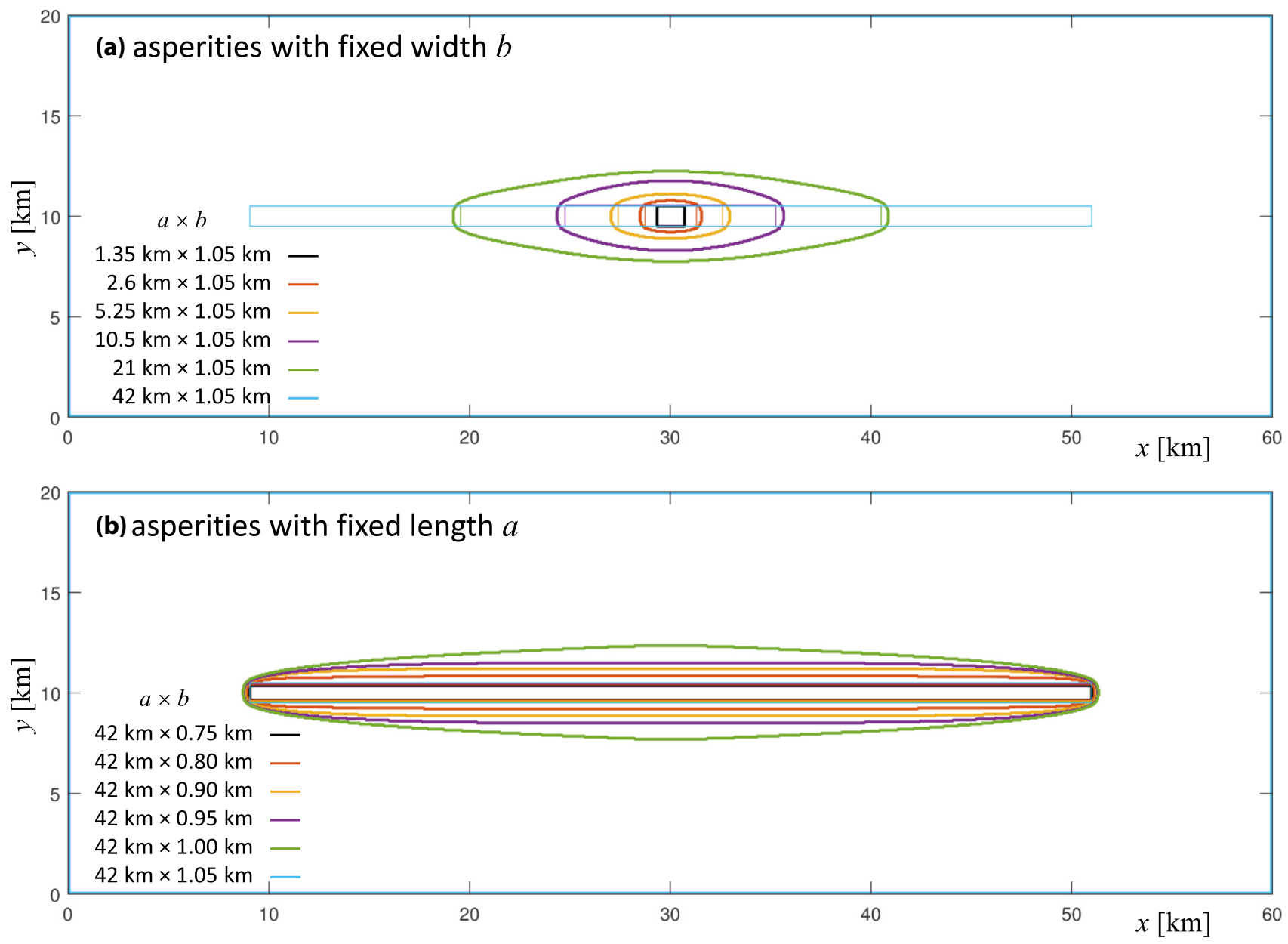

Figure 6. Comparison of ruptures nucleated by asperities with fixed width (a) or fixed length (b). Asperities are depicted by thin lines and contours of the final size of ruptures by bold lines. The light blue frame indicates the runaway rupture nucleated by asperity with $a=42 \mathrm{~km}$ and $b=1.05 \mathrm{~km}$. The changes of width of the asperities in (b) are so small that the asperities are almost indistinguishable.

(a)

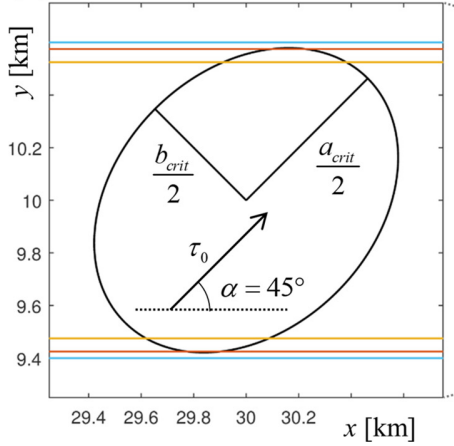

(b)

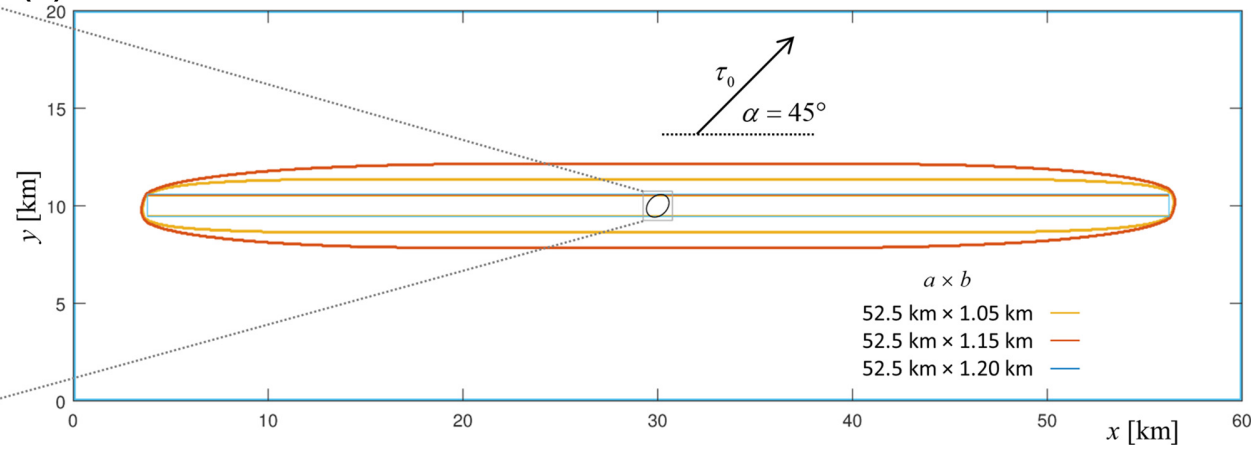

Figure 7. Results of the numerical test for oblique orientation of asperity and initial traction. (a) Visual comparison of width of the asperity with the ellipse determined by the critical lengths ( $a_{\text {crit }}$ and $b_{\text {crit }}$ ) and orientation of the initial traction $\tau_{0}$. (b) Final sizes of ruptures (bold lines) nucleated by the three considered asperities (thin lines).

the third simulation, we set $b=1.2 \mathrm{~km}$. The asperity is now wide enough to include the critical ellipse and, as expected, it produced a runaway rupture.

Although the results of these limited numerical tests are consistent with our expectations, a detailed parametric study, investigating various orientations, aspect ratios and $S$ values is required to confirm our conjectures. We leave such a study for future work.

\section{CONCLUSIONS}

We studied how elongated overstressed asperities hosting earthquake initiation affect rupture nucleation and arrest. Problems with very elongated asperities, that is, with aspect ratio smaller than $\alpha_{\min }$ or greater than $\alpha_{\max }$ (see eq. 1) reduce to a 2-D problem. For low values of strength parameter $S$ (i.e. for high background stress level), the critical length for runaway ruptures is independent of $S$, 
in this case, the large-scale-yielding estimates by Uenishi \& Rice (2003) are sufficient. If $S>2.75$, the critical length depends on $S$, for which we derived small-scale-yielding analytical estimates and verified them against results of numerical simulations. Galis et al. (2015) observed a similar transition in 3-D between largeand small-scale-yielding regimes, but at a lower critical value of $S \sim 0.75$.

Because a theoretical analysis of ruptures nucleated on elongated asperities is not available, we have performed a parametric study using numerical dynamic rupture simulations. We found that the transition to runaway ruptures is controlled by either asperity length or asperity area depending on aspect ratio and background stress. The transition between the area-controlled and length-controlled regimes is narrow. We proposed conditions to determine whether an asperity will produce an arrested or a runaway rupture.

We further investigated the size of arrested ruptures and compared results of numerical simulations with 2-D and 3-D theoretical estimates of rupture arrest size (Ampuero \& Ben-Zion 2008; Galis et al. 2017). Rupture arrest follows three separate regimes depending on asperity aspect ratio and background stress: a length-controlled regime, in which final rupture size is well predicted by 2-D estimates by Ampuero \& Ben-Zion (2008); an area-controlled regime, in which final rupture area follows the 3D estimates by Galis et al. (2017); and a mixed regime in which the rupture arrest is controlled neither by asperity length nor by area. In the last case, no analytical estimate of the final rupture size is available, but an upper bound is provided by Galis et al. (2017).

The core of our analysis focused on asperities aligned with or perpendicular to the initial fault shear traction. Under oblique orientation of stress with respect to the asperity, we hypothesized that runaway ruptures occur if both axes of the asperity-inscribed ellipse with major axis oriented parallel to the initial traction are larger than the critical lengths and its area is larger than the critical area. Results of a limited set of simulations supported this hypothesis.

In the context of induced seismicity, our idealized model results provide scenarios that could be either favourable or challenging for TLSs and allow gaining insights into the mechanical conditions leading to them. Situations with a progressive transition from arrested to runaway ruptures, involving events of growing size over an extended period, constitute the best-case scenario for a TLS. A sudden transition to runaway rupture, a challenging situation for a TLS, may occur on very elongated asperities with shorter dimension close to the critical size for runaway rupture (variations of the longer asperity side have only limited effects).

\section{ACKNOWLEDGEMENTS}

The research reported in this publication was supported by funding from King Abdullah University of Science and Technology (KAUST), grant BAS/1/1339-01-01. MG and JK acknowledge partial support by the Slovak Foundation Grant VEGA$2 / 0188 / 15$. JPA acknowledges partial funding from NAM (Nederlandse Aardolie Maatschappij) and from the French government through the UCA-JEDI Investments in the Future project managed by the National Research Agency (ANR) with the reference number ANR-15-IDEX-01. We appreciate reviews by two anonymous reviewers that helped us to improve the manuscript.

\section{R EFER E N CES}

Ader, T. et al., 2012. Convergence rate across the Nepal Himalaya and interseismic coupling on the Main Himalayan Thrust: Implications for seismic hazard, J. geophys. Res., 117(B4), doi:10.1029/2011JB009071.

Ampuero, J.-P., Vilotte, J.-P. \& Sánchez-Sesma, F.J., 2002. Nucleation of rupture under slip dependent friction law: simple models of fault zone, J. geophys. Res., 107(B12), ESE 2-1-ESE 2-19, doi:10.1029/2001JB000452.

Ampuero, J.P., 2008a. BIMAT-PCSI. Available at: http://web.gps.caltech.ed $\mathrm{u} / \sim$ ampuero/soft/bimat_1.1.2.tar.gz, Access date Feb22, 2019.

Ampuero, J.P., 2008b. SBIEMLAB. Available at: http://web.gps.caltech.ed $\mathrm{u} / \sim$ ampuero/soft/SBIEMLAB_0.1.tar.gz.

Ampuero, J.P. \& Ben-Zion, Y., 2008. Cracks, pulses and macroscopic asymmetry of dynamic rupture on a bimaterial interface with velocityweakening friction, Geophys. J. Int., 173(2), 674-692.

Ampuero, J.P., Ripperger, J. \& Mai, P.M., 2006. Properties of dynamic earthquake ruptures with heterogeneous stress drop, Geophys. Monogr. Ser., 170, 255-261.

Avouac, J.-P., Meng, L., Wei, S., Wang, T. \& Ampuero, J.-P., 2015. Lower edge of locked Main Himalayan Thrust unzipped by the 2015 Gorkha earthquake, Nat. Geosci., 8(9), 708-711.

Azad, M., Garagash, D.I. \& Satish, M., 2017. Nucleation of dynamic slip on a hydraulically fractured fault, J. geophys. Res., 122(4), 2812-2830.

Bruhat, L. \& Segall, P., 2017. Deformation rates in northern Cascadia consistent with slow updip propagation of deep interseismic creep, Geophys. J. Int., 211(1), 427-449.

Buijze, L., van den Bogert, P.A.J., Wassing, B.B.T., Orlic, B. \& ten Veen, J., 2017. Fault reactivation mechanisms and dynamic rupture modelling of depletion-induced seismic events in a Rotliegend gas reservoir, Neth. J. Geosci., 96(05), s131-s148.

Cochard, A. \& Rice, J.R., 2000. Fault rupture between dissimilar materials: Ill-posedness, regularization, and slip-pulse response, Journal of Geophysical Research: Solid Earth. Wiley-Blackwell, 205(B11), 2589125907, doi:10.1029/2000JB900230.

Day, S.M., Dalguer, L.A., Lapusta, N. \& Liu, Y., 2005. Comparison of finite difference and boundary integral solutions to three-dimensional spontaneous rupture, J. geophys. Res., 110(12), 1-23.

Dempsey, D. \& Suckale, J., 2016. Collective properties of injection-induced earthquake sequences: 1 . Model description and directivity bias, J. geophys. Res., 121(5), 3609-3637.

Dunham, E.M., 2007. Conditions governing the occurrence of supershear ruptures under slip-weakening friction, J. geophys. Res., 112(7), 1-24.

Ellsworth, W.L., 2013. Injection-induced earthquakes, Science, 341(6142), doi:10.1126/science.1225942.

Galis, M., Ampuero, J.P., Mai, P.M. \& Cappa, F., 2017. Induced seismicity provides insight into why earthquake ruptures stop, $S c i . A d v$., 3(12), doi:10.1126/sciadv.aap7528.

Galis, M., Pelties, C., Kristek, J., Moczo, P., Ampuero, J.-P. \& Mai, P.M., 2015. On the initiation of sustained slip-weakening ruptures by localized stresses, Geophys. J. Int., 200(2), 888-907.

Garagash, D.I. \& Germanovich, L.N., 2012. Nucleation and arrest of dynamic slip on a pressurized fault, J. geophys. Res., 117(B10), doi:10.1029/2012JB009209.

Kato, N., 2012. Fracture energies at the rupture nucleation points of large interplate earthquakes, Earth planet. Sci. Lett., 353-354, 190-197.

Lapusta, N., Rice, J.R., Ben-Zion, Y. \& Zheng, G., 2000. Elastodynamic analysis for slow tectonic loading with spontaneous rupture episodes on faults with rate- and state-dependent friction, J. geophys. Res., 105(B10), $23765-23789$.

Michel, S., Avouac, J.-P., Lapusta, N. \& Jiang, J., 2017. Pulse-like partial ruptures and high-frequency radiation at creeping-locked transition during megathrust earthquakes, Geophys. Res. Lett., 44(16), 8345-8351.

Mildon, Z.K., Roberts, G.P., Faure Walker, J.P. \& Iezzi, F., 2017. Coulomb stress transfer and fault interaction over millennia on non-planar active normal faults: the $M_{\mathrm{W}} 6.5-5.0$ seismic sequence of 2016-2017, central Italy, Geophys. J. Int., 210(2), 1206-1218.

Moczo, P., Kristek, J. \& Galis, M., 2014. The Finite-Difference Modelling of Earthquake Motions: Waves and Ruptures, Cambridge Univ. Press. 
Morrisey, J.W. \& Geubelle, P.H., 1997. A numerical scheme for mode III dynamic fracture problems, Int. J. Numer. Methods Eng., 40(7), 11811196.

Mulder, F.M.M., 2003. Modelling of Stress Development and Fault Slip in and Around a Producing Gas Reservoir, PhD thesis, Technical University of Delft.

Ripperger, J., Ampuero, J.P., Mai, P.M. \& Giardini, D., 2007. Earthquake source characteristics from dynamic rupture with constrained stochastic fault stress, J. geophys. Res., 112(4), 1-17.

Rubin, A.M. \& Ampuero, J.-P., 2007. Aftershock asymmetry on a bimaterial interface, J. geophys. Res., 112(B5), B05307, doi:10.1029/2006JB004337.

Uenishi, K., 2009. On the mechanical destabilization of a three-dimensional displacement-softening plane of weakness, in Proceedings of the 38th Symposium on Rock Mechanics, Tokyo, Japan, 8-9 January 2009, Committee on Rock Mechanics, pp. 332-337, The Japan Society of Civil Engineers, Tokyo, Japan.

Uenishi, K., 2018. Three-dimensional fracture instability of a displacementweakening planar interface under locally peaked nonuniform loading, $J$. Mech. Phys. Solids, 115, 195-207.

Uenishi, K. \& Rice, J.R., 2003. Universal nucleation length for slipweakening rupture instability under nonuniform fault loading, J. geophys. Res., 108(B1), 2042.

Van Wees, J.-D. et al., 2017. Geomechanical models for induced seismicity in the Netherlands: inferences from simplified analytical, finite element and rupture model approaches, Neth. J. Geosci., 96(05), s183-s202.

\section{APPENDIX A: CRITICAL LENGTHS FOR 2-D RUNAWAY RUPTURES}

Here we derive estimates of critical lengths for runaway ruptures on 2-D faults based on the Griffith crack equilibrium criterion and small-scale-yielding fracture mechanics, valid at low background stress levels. Then, in combination with known large-scale-yielding nucleation lengths, we provide a critical length estimate valid at all stress levels.

Adopting the approach of Galis et al. (2015; Appendix A), Ripperger et al. (2007; Appendix B) and Azad et al. (2017), we assume an overstressed asperity with half-length $a_{i}$ with stress $\tau_{i}$ (greater than the static friction $\tau_{s}$ ). The static stress intensity factor for symmetric non-uniform stress drop is (Ampuero et al. 2006)

$K_{0}(a)=\sqrt{\pi a} \frac{2}{\pi} \int_{0}^{a} \frac{\Delta \tau(x)}{\sqrt{a^{2}-x^{2}}} \mathrm{~d} x$,

where $a$ is the crack half-length and $\Delta \tau$ the stress drop. The criterion for crack arrest is

$\eta K_{0} \leq K_{\mathrm{c}}$,

where $\eta$ is an adjustable factor to account for effects of overshoot (Galis et al. 2015). We calibrate its value numerically (Appendix B). Assuming linear slip-weakening friction, $K_{\mathrm{c}}$ may be approximated as

$K_{\mathrm{c}}^{2}=2 \cdot \mu^{*} G_{\mathrm{c}}=\mu^{*} \cdot D_{\mathrm{c}} \cdot\left(\tau_{\mathrm{s}}-\tau_{\mathrm{d}}\right)$,

where $D_{\mathrm{c}}$ is the characteristic slip-weakening distance, $\tau_{\mathrm{s}}$ and $\tau_{\mathrm{d}}$ are static and dynamic frictional strengths, respectively, and $\mu^{*}=\mu$ for mode III or $\mu^{*}=\mu /(1-v)$ for mode II, where $\mu$ is shear modulus and $v$ is Poisson's ratio.

A first estimate of the critical size for runaway rupture is derived assuming $a_{i}<<a$. The effect of an overstressed asperity on a fault with uniform background stress $\tau_{0}$ can then be represented using a point-load approximation as

$\Delta \tau(x)=\Delta \tau_{0}+F . \delta(x)$, where $\Delta \tau_{0}=\tau_{0}-\tau_{\mathrm{d}}$ is the background stress drop, $\delta(x)$ is the Dirac delta function and $F=\frac{1}{2} a_{i} \cdot\left(\tau_{i}-\tau_{0}\right)$ is the force due to excess stress on the asperity. Eq. (A1) then yields

$K_{0}(a)=\Delta \tau_{0} \sqrt{\pi a}+\frac{2 F}{\sqrt{\pi a}}$.

This function has a single minimum, $K_{0}^{\min }=\sqrt{8 \cdot F \cdot \Delta \tau_{0}}$. The condition for runaway rupture, obtained by negating the condition in eq. (A2), is then

$\eta K_{0}^{\min }>K_{\mathrm{c}}$.

By solving eq. (A6) for $a_{i}$ we find the first-order condition for runaway rupture

$a_{i}>a_{1}^{\prime}=\frac{1}{\eta^{2}} \frac{\mu^{*} \cdot D_{\mathrm{c}} \cdot\left(\tau_{\mathrm{s}}-\tau_{\mathrm{d}}\right)}{8 \cdot\left(\tau_{\mathrm{i}}-\tau_{0}\right) \cdot\left(\tau_{0}-\tau_{\mathrm{d}}\right)}$.

A more accurate estimate is derived now. For arbitrary values of $a_{i}<a$, eq. (A1) yields

$K_{0}(a)=\sqrt{\pi a}\left(\tau_{0}-\tau_{\mathrm{d}}\right)\left[1+\frac{\tau_{i}-\tau_{0}}{\tau_{0}-\tau_{\mathrm{d}}} \frac{2}{\pi} \arcsin \left(\frac{a_{\mathrm{i}}}{a}\right)\right]$.

The condition for runaway rupture, eq. (A6), can no longer be solved analytically for $a_{\mathrm{i}}$. To facilitate numerical solution, we introduce the function

$f(x, \gamma)=\sqrt{x}\left[1+\frac{2 \gamma}{\pi} \arcsin \left(\frac{1}{x}\right)\right]$

and rewrite eq. (A8) as

$K_{0}(a)=\sqrt{\pi a_{\mathrm{i}}}\left(\tau_{0}-\tau_{\mathrm{d}}\right) f\left(a / a_{\mathrm{i}}, \gamma\right)$,

where $\gamma=\left(\tau_{\mathrm{i}}-\tau_{0}\right) /\left(\tau_{0}-\tau_{\mathrm{d}}\right)$. The stress intensity factor has a single minimum

$K_{0}^{\min }=\sqrt{\pi a_{i}}\left(\tau_{0}-\tau_{d}\right) f_{\min }(\gamma)$,

where $f_{\min }(\gamma)=\min _{x} f(x, \gamma)$ is computed numerically. Solving eq. (A6) then yields

$a_{\mathrm{i}}>a_{2}^{\prime}=\frac{1}{\eta^{2}} \frac{\mu^{*} \cdot D_{\mathrm{c}} \cdot\left(\tau_{\mathrm{s}}-\tau_{\mathrm{d}}\right)}{\pi \cdot\left(\tau_{0}-\tau_{\mathrm{d}}\right)^{2} \cdot f_{\min }^{2}(\gamma)}$.

Eqs (A7) and (A12) are derived for half-lengths. However, in the main part of the paper we find it more convenient to work with the full asperity length. We thus define the critical length, in the small-scale yielding regime, as

$a_{2}=2 a_{2}^{\prime}=\frac{2}{\eta^{2}} \frac{\mu^{*} \cdot D_{\mathrm{c}} \cdot\left(\tau_{\mathrm{s}}-\tau_{\mathrm{d}}\right)}{\pi \cdot\left(\tau_{0}-\tau_{\mathrm{d}}\right)^{2} \cdot f_{\min }^{2}(\gamma)}$.

The critical length $a_{2}$ depends on the strength parameter $S=$ $\left(\tau_{s}-\tau_{0}\right) /\left(\tau_{0}-\tau_{d}\right)$. As found in 3-D by Galis et al. (2015) and in 2D by Azad et al. (2017), the estimate $a_{2}$ is valid for low background stress (high values of $S$ ), whereas at high stress (low $S$ ) the critical length is the large-scale-yielding estimate of nucleation size by Uenishi \& Rice (2003)

$a_{\mathrm{UR}} \cong 1.158 \mu^{*} \frac{D_{\mathrm{c}}}{\tau_{\mathrm{s}}-\tau_{\mathrm{d}}}$.

Our final estimate of the critical size for runaway rupture in 2-D, valid for all values of $S$, is

$a_{\mathrm{c}}=\max \left(a_{\mathrm{UR}}, a_{2}\right)$.

Fig. A1 shows this estimate as a function of $S$.

Additionally, Fig. A1 indicates the location of three rupture regimes in parameter space $\left(S, a / L_{\text {fric }}\right)$. The critical length $a_{\mathrm{UR}}$ 


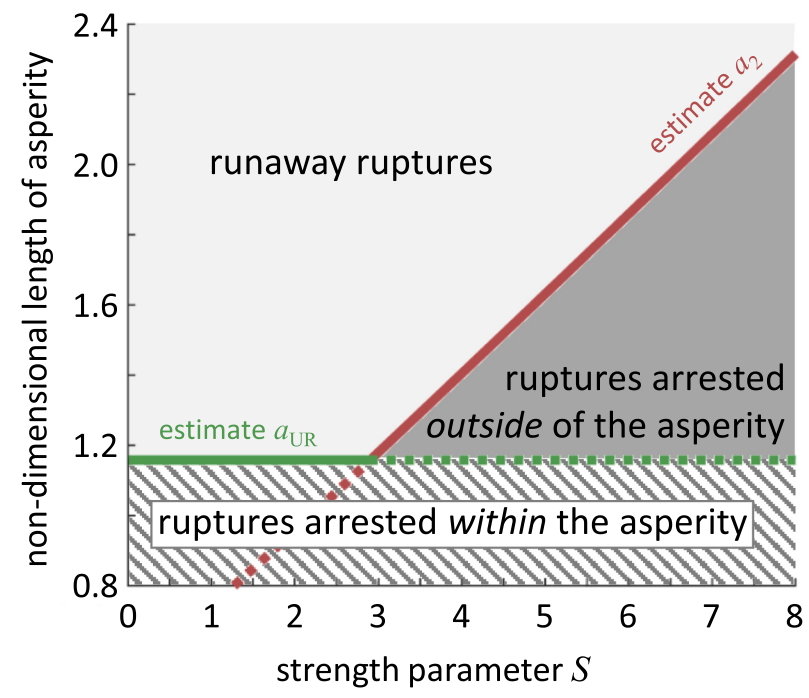

Figure A1. Dependence of critical length $a_{\mathrm{c}}=\max \left(a_{\mathrm{UR}}, a_{2}\right)$ on strength parameter $S$ and different rupture regimes in 2-D mode III rupture. The asperity length is normalized by $L_{\text {fric }}=\mu . D_{\mathrm{c}} /\left(\tau_{\mathrm{s}}-\tau_{\mathrm{d}}\right)$.

by Uenishi $(2009,2018)$ is the minimum asperity length for the onset of slip instability. Asperities smaller than $a_{\mathrm{UR}}$ produce ruptures that stop quickly and remain contained inside the asperity. Asperities larger than $a_{\mathrm{UR}}$ produce runaway ruptures in high background stress (low $S$ ) environments. At low background stress, asperities larger than $a_{\mathrm{UR}}$ produce ruptures that stop spontaneously outside the asperity, and asperities larger than $a_{2}$ produce runaway ruptures.

\section{APPENDIX B: VERIFICATION AND CALIBRATION OF THE THEORETICAL ESTIMATES OF CRITICAL LENGTHS}

Here we summarize results of our numerical tests to determine the value of the adjustable parameter $\eta$ and the regions of applicability of $a_{1}=2 a_{1}^{\prime}, a_{2}$ and $a_{\mathrm{UR}}$ estimates. We utilized two implementations of the spectral boundary integral equation method for 2-D rupture dynamics (Morrisey \& Geubelle 1997; Cochard \& Rice 2000; Lapusta et al. 2000), the BIMAT-PCSI code for mode II rupture (Rubin \& Ampuero 2007; Ampuero 2008a) and the SBIEMLAB code for mode III rupture (Ampuero 2008b). In both cases, we used a spatial discretization of $14.65 \mathrm{~m}$, which is 40 times smaller than the static process zone size $\Lambda_{0}=617 \mathrm{~m}$, which we found to be largely sufficient for proper numerical resolution of dynamic rupture problems with slip-weakening friction (Day et al. 2005). To accommodate varying strength parameter $S$, we considered fixed values of static and dynamic frictional strength, $\tau_{\mathrm{s}}$ and $\tau_{\mathrm{d}}$, and variable initial shear traction $\tau_{0}$. All parameter values are summarized in Table B1. Additionally, we considered three overstress levels in the asperity-low, medium and high, corresponding to $0.00005,0.5$ and 5 per cent of the strength excess, $\left(\tau_{s}-\tau_{0}\right)$, respectively.

The results of our numerical simulations are summarized and compared with the theoretical estimates in Fig. B1. First, we discuss the cases of low and medium overstress, which lead to very similar results. For $S>2.5$, the numerical results are consistent with the theoretical estimates $a_{1}$ and $a_{2}$, whereas for $S \leq 2.5$ they are consistent with the estimate $a_{\mathrm{UR}}$. These results confirm the applicability of the estimate of the critical length $a_{\mathrm{c}}$ given in eq. (A15). We also confirm that the $S$-value at which the critical asperity size becomes $S$ dependent $(S=2.87)$ is greater than in $3-\mathrm{D}(S \approx 0.75)$. For high overstress and $S>2$, our estimates $a_{1}$ and $a_{2}$ are consistent with the numerical results. However, for $S<2$ the critical length obtained numerically is smaller than $a_{\mathrm{UR}}$. The reason is that the estimate $a_{\mathrm{UR}}$ has been derived without considering overstress. This deviation is thus expected, and it is consistent with $3-\mathrm{D}$ results presented by Galis et al. (2015). Although the overstress is relatively high, 5 per cent of the strength excess, it varies only from $80 \mathrm{kPa}$ for $S=0.1$ to $600 \mathrm{kPa}$ for $S=2$, which are values within the expected range of effects of pore pressure in the context of induced seismicity.

We used the comparison of the theoretical estimates $a_{1}$ and $a_{2}$ with numerical results also to calibrate the adjustable factor $\eta$. Through trial-and-error we find that $\eta=1.04\left(1 / \eta^{2}=0.925\right)$ yields consistent results for mode II and III ruptures in the considered range of overstress levels.

The value of $S$ at the transition between small- and large-scaleyielding regimes depends slightly on the overstress (Fig. B1). The exact value can be obtained from the condition $a_{2}=a_{\mathrm{UR}}$. Although the exact value for low to medium overstress is $S=2.87$, numerical results suggest (Fig. 1b) that it ranges from 2.5 to 3 . Therefore, in the main text we refer to the transition value as $S \approx 2.75$. Galis et al. (2015) observed qualitatively the same transition in 3-D, but at a lower value $S \approx 0.75$, obtained from the condition $A_{2}=A_{\mathrm{U}}$. 
Table B1. Summary of parameter values used for the numerical simulations; $\sigma_{0}$ —initial normal traction, $\tau_{0}$ —initial shear traction (varied to satisfy the desired value of $S$ ), $S$-strength parameter, $\mu_{\mathrm{s}}$ —static friction coefficient, $\mu_{\mathrm{d}}$-dynamic friction coefficient, $D_{\mathrm{c}}$-characteristic slip-weakening distance, $v_{\mathrm{s}}-$ shear wave speed and $\rho$-density.

\begin{tabular}{lccccccc}
\hline$\sigma_{0}$ & $\tau_{0}$ & $S$ & $\mu_{\mathrm{s}}$ & $\mu_{\mathrm{d}}$ & $D_{\mathrm{c}}$ & $v_{\mathrm{s}}$ & $\rho$ \\
\hline $120 \mathrm{MPa}$ & $65.619-81.152 \mathrm{MPa}$ & $0.1-6.0$ & 0.6778 & 0.525 & $0.4 \mathrm{~m}$ & & $3464 \mathrm{~m} \mathrm{~s}^{-1 \mathrm{TH}}$ \\
\hline
\end{tabular}
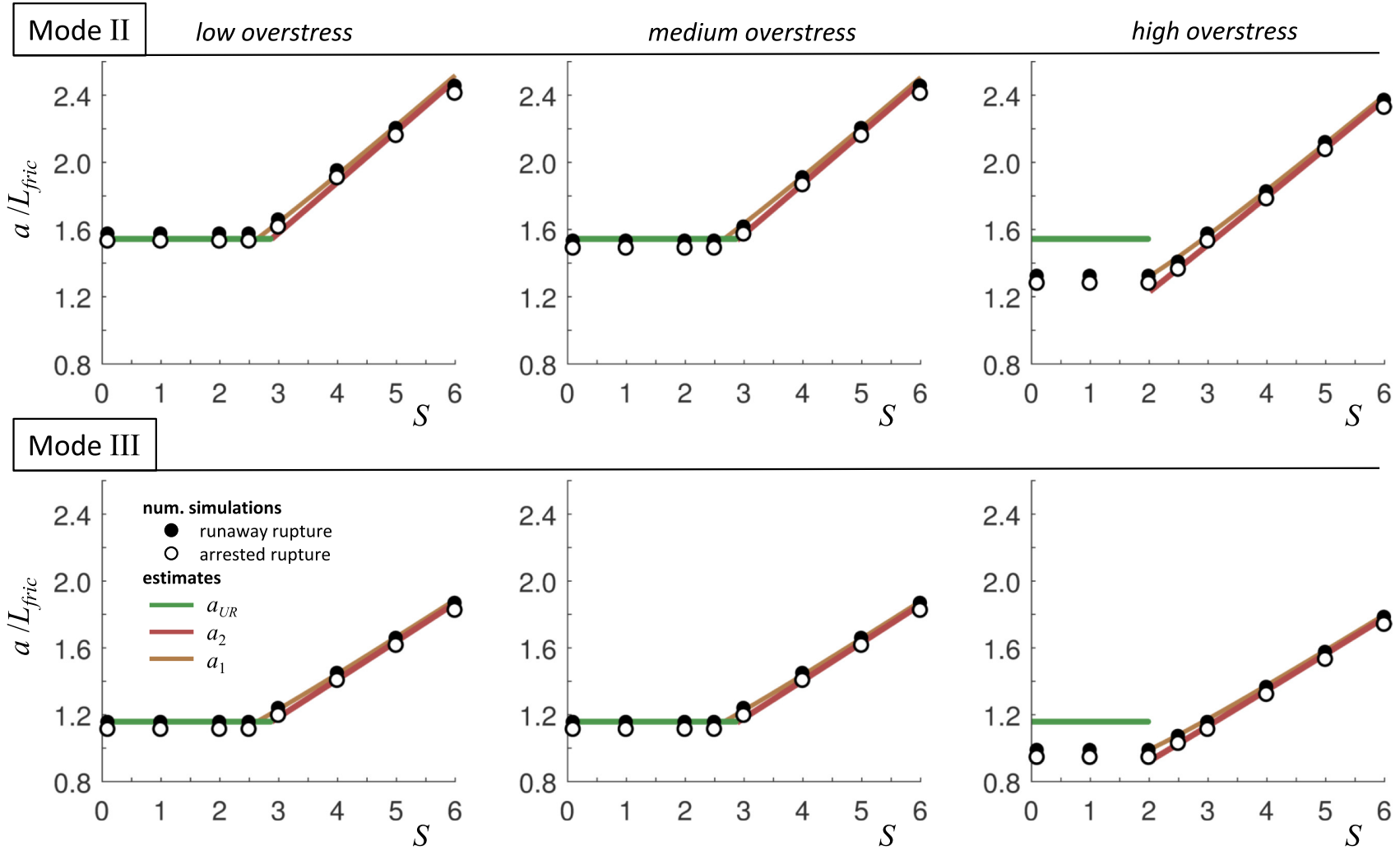

Figure B1. Comparison of the transition to the runaway ruptures observed in numerical simulations (depicted by symbols) with theoretical estimates (depicted by lines) for mode II and mode III ruptures and for three considered levels of the overstress- low $(0.0000005$ per cent of the strength excess), medium $(0.5$ per cent of the s.e.) and high (5 per cent of the s.e.). The results are plotted in terms of dimensionless length of the asperity $a / L_{\text {fric }}\left(\right.$ where $\left.L_{\text {fric }}=\mu . D_{\mathrm{c}} /\left(\tau_{\mathrm{s}}-\tau_{\mathrm{d}}\right)\right)$ as a function of the strength parameter $S$.

\section{APPENDIX C: SUMMARY OF \\ CONDITIONS FOR RUNWAY RUPTURE \\ AND RUPTURE ARREST}

This appendix contains only Tables $\mathrm{C} 1$ and $\mathrm{C} 2$. 
Table C1. Summary of conditions for runaway rupture in 2-D and 3-D; $\mu$ is shear modulus, $\nu$ is Poisson's ratio, $\tau_{\mathrm{s}}, \tau_{\mathrm{d}}$ — static and dynamic frictional strengths, $D_{\mathrm{c}}$ —characteristic slip-weakening distance, $\tau_{0}$ —initial shear traction outside of an asperity and $\tau_{i}$ —initial shear traction inside of an asperity.

\section{Conditions for runaway rupture}

In 2D - critical crack-length for mode II $\left(\mu^{*}=\mu /(1-v)\right)$ and mode III $\left(\mu^{*}=\mu\right)$

\begin{tabular}{|c|c|}
\hline \multicolumn{2}{|l|}{$a_{c r i t}=\max \left(a_{U R}, a_{2}\right)$} \\
\hline$a_{U R} \cong 1.158 \frac{\mu^{*} \cdot D_{c}}{\tau_{s}-\tau_{d}}$ & Uenishi \& Rice (2003) \\
\hline
\end{tabular}

where $f_{\min }$ is the minimum of the function

$f(x)=\sqrt{x}\left[1+\frac{2}{\pi} \frac{\tau_{i}-\tau_{0}}{\tau_{0}-\tau_{d}} \arcsin \left(\frac{1}{x}\right)\right]$

Appendices $A$ and $B$

In 3D - critical area for an asperity with aspect ratio close to one

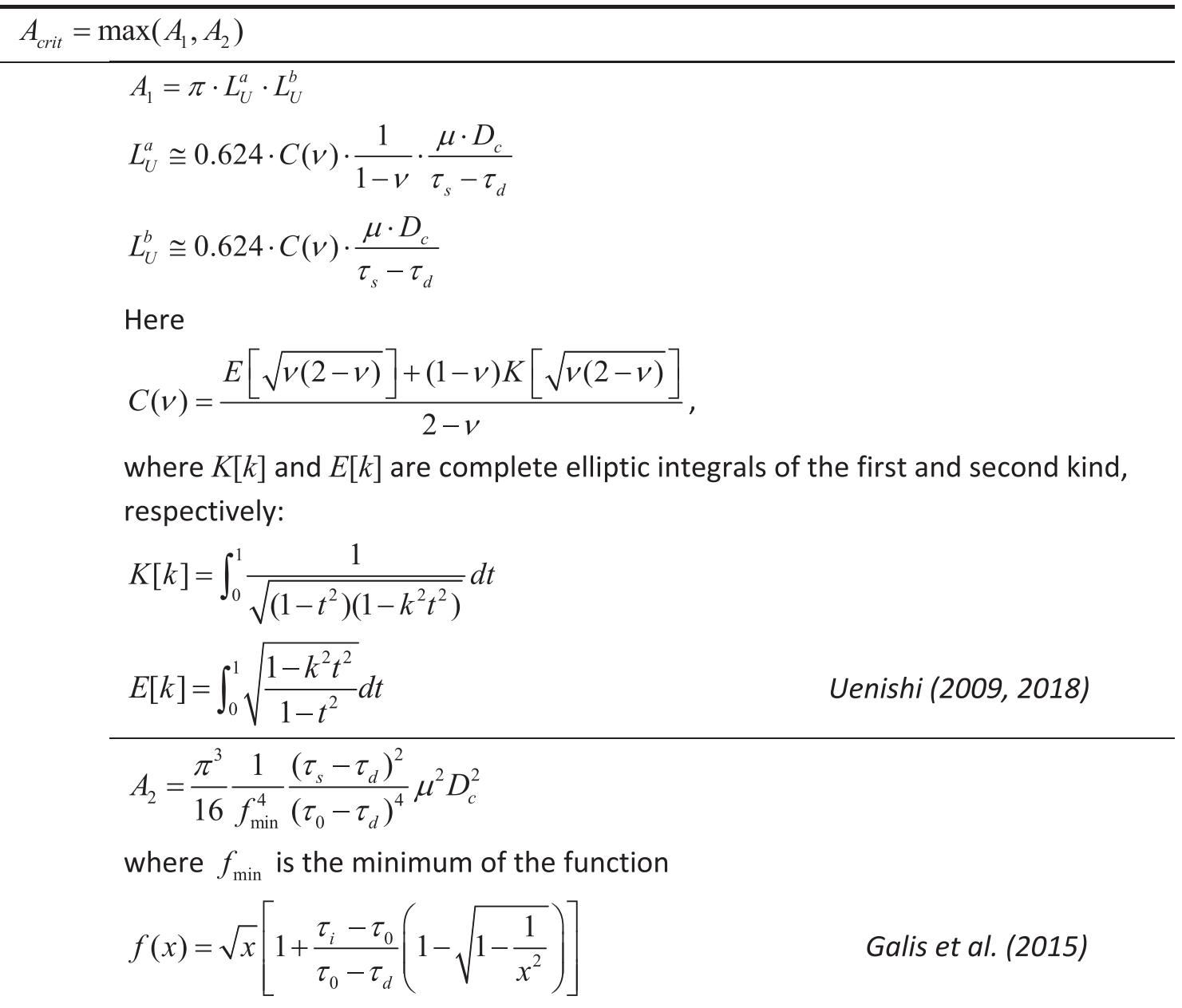


Table C2. Summary of conditions for rupture arrest for 2-D and 3-D problems; $\mu$ is shear modulus, $\tau_{\mathrm{s}}, \tau_{\mathrm{d}}$ - static and dynamic frictional strengths, $D_{\mathrm{c}}-$ characteristic slip-weakening distance, $\tau_{0}$ —initial traction outside an asperity, $\tau_{\mathrm{i}}$-initial traction inside an asperity, $a_{\mathrm{i}}$ —half-length or radius of an asperity, for 2-D and 3-D, respectively.

\section{Conditions for rupture arrest and estimation of size of arrested rupture}

Conditions for rupture arrest

$$
K_{0}(R)=K_{c} \quad \text { and } \quad \frac{\partial\left(K_{0}-K_{c}\right)}{\partial R}<0
$$

Solving both conditions simultaneously for $R$ yields rupture size at the time of arrest. The rupture size is crack half-length or crack radius, for 2D or 3D problem, respectively.

The conditions are usually solved numerically because an analytical solution may not be available for considered form of static stress intensity factor, $K_{0}$, and/or rupture toughness, $K_{c}$.

Galis et al (2017)

\section{Rupture toughness, $K_{c}$}

$$
K_{c}=\sqrt{2 \mu \cdot G_{c}}
$$

where $G_{c}$ is fracture energy.

For linear-slip weakening

$$
K_{c}=\sqrt{\mu \cdot D_{c} \cdot\left(\tau_{s}-\tau_{d}\right)}
$$

\section{Static stress intensity factor, $K_{0}$}

In 2D - for an asperity with half-length $a_{i}$

$$
K_{0}(R)=\sqrt{\pi R}\left(\tau_{0}-\tau_{d}\right)\left[1+\frac{2}{\pi} \frac{\tau_{i}-\tau_{0}}{\tau_{0}-\tau_{d}} \arcsin \left(\frac{a_{i}}{R}\right)\right]
$$

Ampuero \& Ben-Zion (2008)

$$
\frac{\text { In 3D - for a circular asperity with radius } a_{i}}{K_{0}(R)=2 \sqrt{\frac{R}{\pi}}\left(\tau_{0}-\tau_{d}\right)\left[1+\frac{\tau_{i}-\tau_{d}}{\tau_{0}-\tau_{d}}\left(1-\sqrt{1-\left(\frac{a_{i}}{R}\right)^{2}}\right)\right]}
$$

